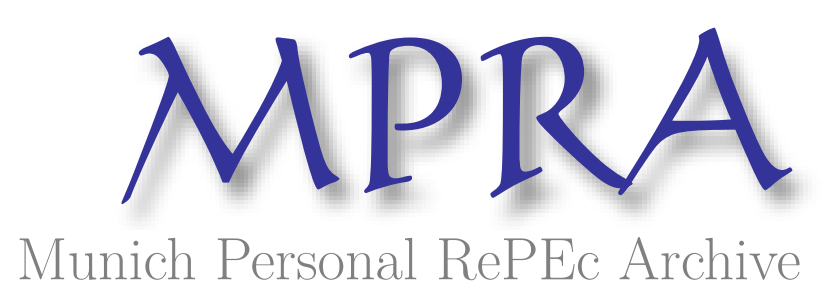

\title{
Path-conditional forward volatility
}

Carey, Alexander

28 February 2006

Online at https://mpra.ub.uni-muenchen.de/4964/

MPRA Paper No. 4964, posted 18 Sep 2007 UTC 


\title{
PATH-CONDITIONAL FORWARD VOLATILITY
}

\author{
Alexander Carey* \\ 4 avenue de la Guillemotte \\ 78112 Fourqueux, France \\ alex.carey@wanadoo.fr
}

February 28, 2006

\begin{abstract}
* Alexander Carey is a graduate of Cass Business School, London. He has worked for Salomon Smith Barney New Zealand.
\end{abstract}

In derivatives modelling, it has often been necessary to make assumptions about the volatility of the underlying variable over the life of the contract. This can involve specifying an exact trajectory, as in the Black and Scholes (1973), Merton (1973) or Black (1976) models; one that depends on the level of the underlying variable as in the local volatility models of Dupire (1994), Derman and Kani (1994) and Rubinstein (1994); or fixing the parameters of a more general stochastic volatility process as in Hull and White (1987) or Heston (1993). These forward-looking assumptions are by their very nature destined to be disproved, and what is more are at odds with the frequent model recalibration that (rightly) takes place in practice.

In Carey (2005), the Black-Scholes analytical framework is extended, via the definition of higher-order volatilities and the derivation of moment formulae for the case where they are deterministic. In this paper, we show that the same formulae can be obtained under markedly weaker assumptions, which leave the future volatilities unspecified. Instead, we impose constraints on new, related quantities, which we term "path-conditional forward volatilities". Under this scheme, the model inputs are no longer 
the future spot volatilities, but rather their forward counterparts. One consequence, we show, is that contrary to conventional wisdom, the Black-Scholes formula can in principle be used without any reference to future volatility.

\section{1. $j$-TH ORDER PATH-CONDITIONAL FORWARD VOLATILITY}

We begin with some preliminary definitions and notation. Let $\left(X_{t}\right)$ be a positivevalued adapted stochastic process on a filtered probability space $\left(\Omega, \mathcal{F},\left(\mathcal{F}_{t}\right), Q\right)$. Let $\delta t>0$ denote a finite period of time, and define $\delta X_{t}=X_{t+\delta t}-X_{t}$, so that the relative change of the process over the interval $t$ to $t+\delta t$ reads $\delta X_{t} / X_{t}$. Let $\mathcal{X}_{t, t^{\prime}}$ denote the $\boldsymbol{\sigma}$-algebra generated by the process over the interval $\left(t, t^{\prime}\right]$, and write $\mathcal{F}_{t} \vee \mathcal{X}_{t, t^{\prime}}$ for the $\boldsymbol{\sigma}$-algebra generated by the union of $\mathcal{F}_{t}$ and $\mathcal{X}_{t, t^{\prime}} \cdot E_{t} \cdot$ denotes expectation conditional on $\mathcal{F}_{t}, E_{t, t^{\prime}}$. denotes expectation conditional on $\mathcal{F}_{t} \vee \mathcal{X}_{t, t^{\prime}}$, and $j$ is a generic positive integer.

Consider an agreement by which two parties undertake to exchange the amount $\left(\delta X_{t^{\prime}} / X_{t^{\prime}}\right)^{j}$, as yet unknown but to be revealed imminently, and a predetermined amount which we tentatively write in the form $\left(\Sigma_{j, t, t^{\prime}}\right)^{j} \times \delta t, t \leq t^{\prime}$. We suppose that the parties are in possession of the information $\mathcal{F}_{t}$, and that given this information, the agreement is a fair gamble under the probability measure $Q$, by which we mean:

$$
E_{t}\left[\left(\delta X_{t^{\prime}} / X_{t^{\prime}}\right)^{j}-\Sigma_{j, t, t^{\prime}}^{j} \delta t\right]=0
$$

This leads us to formally define the quantity $\Sigma_{j, t, t^{\prime}}$ via the identity:

$$
\Sigma_{j, t, t^{\prime}}^{j} \equiv E_{t}\left(\delta X_{t^{\prime}} / X_{t^{\prime}}\right)^{j} / \delta t
$$


with the convention that $\Sigma_{j, t, t^{\prime}}$ equals the nonnegative root when $j$ is even. We shall refer to $\Sigma_{j, t, t^{\prime}}$ as $j$-th order finite-period forward volatility. Of particular interest will be the limit of vanishingly small $\delta t$, for which we define the quantity $\sigma_{j, t, t^{\prime}}$ via:

$$
\sigma_{j, t, t^{\prime}}^{j} \equiv \lim _{\delta t \backslash 0} \Sigma_{j, t, t^{\prime}}^{j}
$$

again with $\sigma_{j, t, t^{\prime}}$ nonnegative when $j$ is even. Equivalently, $\sigma_{j, t, t^{\prime}}=\lim _{\delta t \backslash 0} \Sigma_{j, t, t^{\prime}}$. We shall refer to $\sigma_{j, t, t^{\prime}}$ as $j$-th order instantaneous forward volatility. When $t^{\prime}=t$ the qualification "forward" will be replaced by "spot", and for this case a prior definition, as well as a rationale for using $j$ as an exponent, can be found in Carey (2005).

Consider next a variation on this scenario under which the exposure $\left(\delta X_{t^{\prime}} / X_{t^{\prime}}\right)^{j}$ is again to be exchanged for a pre-agreed premium, but the information available to the parties is no longer $\mathcal{F}_{t}$, but $\mathcal{F}_{t} \vee \mathcal{X}_{t, t^{\prime}}$. Heuristically, in addition to the information $\mathcal{F}_{t}$, the parties know the path followed by the process in the interim between times $t$ and $t^{\prime}$. Writing the new premium as $\left(\hat{\Sigma}_{j, t, t^{\prime}}\right)^{j} \times \delta t$, we again suppose that the agreement is a fair gamble under $Q$, that is:

$$
E_{t, t^{\prime}}\left[\left(\delta X_{t^{\prime}} / X_{t^{\prime}}\right)^{j}-\hat{\Sigma}_{j, t, t^{\prime}}^{j} \delta t\right]=0
$$

We thus define the quantity $\hat{\Sigma}_{j, t, t^{\prime}}$ via the identity:

$$
\hat{\Sigma}_{j, t, t^{\prime}}^{j} \equiv E_{t, t^{\prime}}\left(\delta X_{t^{\prime}} / X_{t^{\prime}}\right)^{j} / \delta t
$$

with the convention that $\hat{\Sigma}_{j, t, t^{\prime}}$ equals the nonnegative root when $j$ is even. We shall refer to $\hat{\Sigma}_{j, t, t^{\prime}}$ as $j$-th order finite-period path-conditional forward volatility. As above, we define the quantity $\hat{\sigma}_{j, t, t^{\prime}}$ via:

$$
\hat{\sigma}_{j, t, t^{\prime}}^{j} \equiv \lim _{\delta t \backslash 0} \hat{\Sigma}_{j, t, t^{\prime}}^{j},
$$


again with $\hat{\sigma}_{j, t, t^{\prime}}$ nonnegative when $j$ is even. Equivalently, $\hat{\sigma}_{j, t, t^{\prime}}=\lim _{\delta t \backslash 0} \hat{\Sigma}_{j, t, t^{\prime}}$. We shall refer to $\hat{\sigma}_{j, t, t^{\prime}}$ as $j$-th order instantaneous path-conditional forward volatility.

For future reference, we make note of the following relationships between the spot volatilities and their forward and path-dependent forward counterparts. Noting that $\mathcal{F}_{t} \subseteq \mathcal{F}_{t^{\prime}}$ and $\mathcal{F}_{t} \vee \mathcal{X}_{t, t^{\prime}} \subseteq \mathcal{F}_{t^{\prime}}$, by the law of iterated expectations we have:

$$
\Sigma_{j, t, t^{\prime}}^{j}=E_{t} \Sigma_{j, t^{\prime}, t^{\prime}}^{j} \text { and } \hat{\Sigma}_{j, t, t^{\prime}}^{j}=E_{t, t^{\prime}} \Sigma_{j, t^{\prime}, t^{\prime \prime}}^{j}
$$

$t^{\prime}>t$ and $\delta t>0$. Analogous results hold for the corresponding instantaneous volatilities, subject to broad technical conditions.

\section{MOMENTS}

We now specialise to the case where finite-period path-conditional forward volatility equals forward volatility, that is:

$$
\hat{\Sigma}_{j, t, t^{\prime}}=\Sigma_{j, t, t^{\prime}}
$$

$t^{\prime}>t$. Heuristically, path-conditional forward volatility is the same for every interim path (more on this later). As shown in the appendix, the $n$-th moment of the process conditional on $\mathcal{F}_{t}$ can then be obtained as:

$$
E_{t} X_{t+\tau}^{n}=X_{t}^{n} \prod_{u}\left[1+\sum_{j=1}^{n}\left(\begin{array}{l}
n \\
j
\end{array}\right) \Sigma_{j, t, u}^{j} \delta t\right],
$$

for $\tau$ a positive multiple of $\delta t$, where the product is over $u=t, t+\delta t, \ldots, t+\tau-\delta t$. If we next suppose that there exists an $\varepsilon>0$ such that (2) holds for every period size $\delta t \leq \varepsilon$, then clearly the instantaneous path-conditional forward volatilities, when they 
exist, also equal their forward counterparts, that is:

$$
\hat{\sigma}_{j, t, t^{\prime}}=\sigma_{j, t, t^{\prime}}
$$

$t^{\prime}>t$. Further, fixing $\tau>0$ and taking the limit $\delta t \searrow 0$ in (3) yields:

$$
E_{t} X_{t+\tau}^{n}=X_{t}^{n} \exp \left[\tau \sum_{j=1}^{n}\left(\begin{array}{l}
n \\
j
\end{array}\right) \sigma_{j}^{j}\right]
$$

where the $\sigma_{j}$, which are assumed to exist, are defined via:

$$
\sigma_{j}^{j} \equiv \frac{1}{\tau} \int_{t}^{t+\tau} \sigma_{j, t, u}^{j} d u,
$$

again with the convention that $\sigma_{j}$ is nonnegative when $j$ is even. We shall refer to $\sigma_{j}$ as $j$-th order average forward volatility. We note here that these moment formulae are the same as those derived in Carey (2005), but with the average volatilities replaced with their forward counterparts. Notably, their derivation has not required any assumption

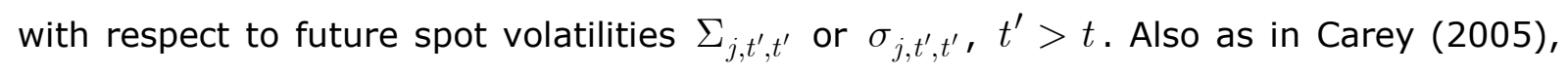
we note that we have not shown that (4) implies (5), if indeed this holds true.

\section{OPTION PRICING}

We now consider a general option pricing implementation. Suppose that $Q$ is an equivalent martingale measure, $\left(\mathcal{F}_{t}\right)$ is the market information structure and $\left(X_{t}\right)$ is a positive-valued adapted market process. For the sake of both simplicity and generality we suppose that $\left(X_{t}\right)$ is a martingale under $Q-$ for example, the forward price of an asset under the forward measure - although this is not mandatory. In this case, firstorder finite-period spot volatility is identically zero, and so by (1) we have 
$\hat{\Sigma}_{1, t, t^{\prime}}=\Sigma_{1, t, t^{\prime}}=\Sigma_{1, t^{\prime}, t^{\prime}}=0, t^{\prime}>t$. That is, first-order finite-period forward and pathconditional forward volatilities are also zero, and condition (2) is satisfied for any interval size $\delta t$. The corresponding instantaneous volatilities follow suit, with $\hat{\sigma}_{1, t, t^{\prime}}=\sigma_{1, t, t^{\prime}}=\sigma_{1, t^{\prime}, t^{\prime}}=0, t^{\prime}>t$.

For an operational implementation, on the basis of the previous section we may fix $t$ and a small interval size $\varepsilon$, and assume that (2) holds for every $\delta t \leq \varepsilon$ for a suitable number of higher orders of $j$. The moments of the market variable are then given by (5), and the average forward volatilities can be implied from the market prices of liquid vanilla options, to be used as risk metrics or as inputs into a pricing scheme. An example of implied values for the S\&P 500 options market can be found in Carey (2005). We again emphasise that no assumptions have been made regarding future spot volatilities $\Sigma_{j, t^{\prime}, t^{\prime}}$ or $\sigma_{j, t^{\prime}, t^{\prime}}, t^{\prime}>t, j \geq 2$.

To obtain Black-Scholes and related formulae, it is enough to make the additional assumption that the average forward volatilities of orders $j>2$ are zero, whereupon the moments (5) define the market variable as lognormal, and in our case Black's (1976) formula applies with second-order average forward volatility $\sigma_{2}$ in the role of the traditional volatility parameter. One rationale could involve noting that, over a suitably small period of time, changes in the market variable are likely to be small, with $\left|\delta X_{t^{\prime}} / X_{t^{\prime}}\right| \ll 1$ almost certainly. Thus, as $j$ increases, the quantity $\Sigma_{j, t, t^{\prime}}^{j}-$ and by extension its limit $\sigma_{j, t, t^{\prime}}^{j}$ - might be assumed to rapidly decrease in magnitude, providing a basis for truncating the sum in (5) to yield the approximation:

$$
E_{t} X_{t+\tau}^{n} \approx X_{t}^{n} e^{\frac{1}{2} n(n-1) \sigma_{2}^{2} \tau},
$$

which again is tantamount to approximating the distribution of the market variable by the lognormal underlying Black's formula. 
Now the assumption that the path-conditional forward volatilities are invariant to the interim path may not be entirely realistic. Indeed, it could be supposed that for even $j$, the exposure $\left(\delta X_{t^{\prime}} / X_{t^{\prime}}\right)^{j}$ should command a higher premium in the case of an erratic, volatile path in the run-up to time $t^{\prime}$, than in the case of a more subdued trajectory. However, for odd $j$ the picture is not as clear, and assumption (2) is possibly less detrimental. Moreover, it is clearly much preferable to assuming that the spot volatilities are deterministic, as in Carey (2005). In the latter case, one is assuming that the premium is invariant not only to the path of the underlying variable over $\left(t, t^{\prime}\right]$, but also to the evolution of its fundamentals, be it developments in related markets, in the wider economy, or in the political environment, all carried in the larger information set $\mathcal{F}_{t^{\prime}}$. In contrast, the weaker assumption (2) allows the spot volatilities to respond and adjust to some measure of new information.

\section{CONCLUSION}

We have introduced the concept of path-conditional forward volatility, and considered the case where it equals forward volatility. This was shown to yield moment formulae analogous to those in Carey (2005), but without any assumptions with respect to future spot volatilities. In particular, Black-Scholes-related formulae were obtained without assuming that volatility is deterministic.

We briefly mention a possible alternative interpretation of path-conditional forward volatility. Our argument featuring $\mathcal{F}_{t} \vee \mathcal{X}_{t, t^{\prime}}$ as an information set, while squarely in the tradition of the gambling analogies used in probability theory, conjures up visions of being locked up in a basement from time $t$, with only a price ticker for company. A more appealing alternative could involve a scenario under which the 
available information remains limited to $\mathcal{F}_{t}$, but the parties agree to condition the exchange of payments on the realisation of some pre-specified interim path. While this works well when $\mathcal{F}_{t}$ and $\mathcal{X}_{t, t^{\prime}}$ are generated by finite partitions, we have eschewed this interpretation owing to the technical idiosyncrasies of the general case.

Another issue to investigate is whether the local volatility models of Dupire (1994), Derman and Kani (1994) and Rubinstein (1994) can be extended along similar lines. An often-voiced criticism of this class of models is that in practice, future spot volatility is constrained in an unrealistic fashion. It may be that a (suitably specified) path-conditional forward volatility can be substituted for the deterministic local volatility function, thereby addressing this issue, and perhaps also offering a more compelling interpretation of empirical implied volatility surfaces. 


\section{APPENDIX}

Following Carey (2005), we note that:

$$
\left(X_{t+\tau} / X_{t}\right)^{n}=\prod_{u}\left(1+\delta X_{u} / X_{u}\right)^{n}=\prod_{u}\left[1+\sum_{j=1}^{n}\left(\begin{array}{c}
n \\
j
\end{array}\right)\left(\delta X_{u} / X_{u}\right)^{j}\right]
$$

using the binomial theorem. Now since:

$$
\mathcal{F}_{t}=\mathcal{F}_{t} \vee \mathcal{X}_{t, t} \subseteq \mathcal{F}_{t} \vee \mathcal{X}_{t, t+\delta t} \subseteq \ldots \subseteq \mathcal{F}_{t} \vee \mathcal{X}_{t, t+\tau-\delta t}
$$

we have:

$$
E_{t} \cdot=E_{t, t} \cdot=E_{t, t} E_{t, t+\delta t} \cdots E_{t, t+\tau-\delta t} \cdot
$$

by the law of iterated expectations. Thus, assuming that the $\hat{\Sigma}_{j, t, u}$ exist and satisfy $\hat{\Sigma}_{j, t, u}=\Sigma_{j, t, u}$ for every $j \leq n$, taking expectations at time $t$ in (A1), applying (A2) and simplifying iteratively yields (3). To derive (5) we note that:

$$
1+\sum_{j=1}^{n}\left(\begin{array}{c}
n \\
j
\end{array}\right) \Sigma_{j, t, u}^{j} \delta t=\exp \left[o(\delta t)+\sum_{j=1}^{n}\left(\begin{array}{c}
n \\
j
\end{array}\right) \Sigma_{j, t, u}^{j} \delta t\right]
$$

where $o(\delta t)$ represents terms which vanish with $\delta t$ faster than $\delta t$ (that is, $o(\delta t) / \delta t \rightarrow 0$ as $\delta t \searrow 0)$. Replacing in (3), we obtain:

$$
E_{t}\left(X_{t+\tau} / X_{t}\right)^{n}=\exp \sum_{u}\left[o(\delta t)+\sum_{j=1}^{n}\left(\begin{array}{c}
n \\
j
\end{array}\right) \Sigma_{j, t, u}^{j} \delta t\right]
$$

Now by definition $\Sigma_{j, t, u}^{j} \rightarrow \sigma_{j, t, u}^{j}$ as $\delta t \searrow 0$, hence $\sum_{u} \Sigma_{j, t, u}^{j} \delta t \rightarrow \sigma_{j}^{j} \tau$, and since $\sum_{u} o(\delta t)=o(\delta t) \tau / \delta t \rightarrow 0$, taking limits in (A3) yields (5). 


\section{REFERENCES}

Black, F. (1976) The pricing of commodity contracts, Journal of Financial Economics 3(1), 167-179.

Black, F. and M. Scholes (1973) The pricing of options and corporate liabilities, Journal of Political Economy 81(3), 637-654.

Carey, A. (2005) Higher-order volatility, working paper, SSRN eLibrary.

http://ssrn.com/abstract $=864084$

Derman, E. and I. Kani (1994) Riding on a smile, Risk 7(2), 32-39.

Dupire, B. (1994) Pricing with a smile, Risk 7(1), 18-20.

Heston, S. (1993) A closed-form solution for options with stochastic volatility with applications to bond and currency options, Review of Financial Studies 6(2), 327-343.

Hull, J. and A. White (1987) The pricing of options on assets with stochastic volatilities, Journal of Finance 42(2), 281-300.

Merton, R. (1973) Theory of rational option pricing, Bell Journal of Economics and Management Science 4(1), 141-183.

Rubinstein, M. (1994) Implied binomial trees, Journal of Finance 49(3), 771-818. 